\title{
Femtosecond Intersystem Crossing in the DNA Nucleobase Cytosine
}

\author{
Martin Richter, † Philipp Marquetand,,$^{\ddagger}$ Jesús González-Vazquez, " Ignacio Sola, ॠ \\ and Leticia González \\ $\dagger$ Institute of Physical Chemistry, Friedrich Schiller University Jena, Helmholtzweg 4, 07743 \\ Jena, Germany \\ $\ddagger$ Institute of Theoretical Chemistry, University of Vienna, Währinger Str. 17, 1090 \\ Vienna, Austria \\ ๆDepartamento de Química Física I, Universidad Complutense, 28040 Madrid, Spain \\ E-mail: leticia.gonzalez@univie.ac.at
}

Keywords: DNA photostability, excited state dynamics, intersystem crossing, spin-orbit coupling, conical intersection

\section{Abstract}

Ab initio molecular dynamics including nonadiabatic and spin-orbit couplings on equal footing is used to unravel the deactivation of cytosine after UV light absorption. Intersystem crossing (ISC) is found to compete directly with internal conversion in tens of femtoseconds, thus making cytosine the organic compound with the fastest triplet population calculated so far. It is found that close degeneracy between singlet and triplet states can more than compensate for very small spin-orbit couplings, leading to efficient ISC. The femtosecond nature of the intersystem crossing process highlights its importance in photochemistry and challenges the conventional view that large singlet-triplet couplings are required for an efficient population flow into triplet states. These findings are important to understand DNA photostability and the photochemistry and dynamics of organic molecules in general.

\section{Graphical TOC Entry}

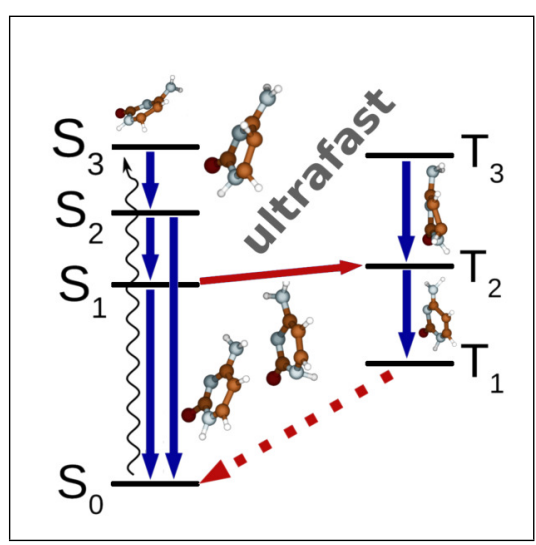


The interaction of DNA and RNA with radiation, from mobile-phone emissions ${ }^{1}$ to $\mathrm{UV}$ wavelengths,, 2 has enthralled the scientific community for years due to its implications in photodamage. ${ }^{3}$ Of particular interest is to understand photostability, i.e. the relaxation mechanisms that bring $\mathrm{DNA}^{4} \sqrt{6}$ to the ground state before any other photoreaction can occur. This means that, instead of fluorescence or phosphorescence, the electronic energy provided upon photoexcitation in DNA is transferred to the nuclear degrees of freedom of the molecular system in different ways. It is precisely the atomistic description of these different relaxation pathways that is still heavily discussed in the literature. In the last years it has been clearly established that excited states of isolated DNA nucleobases undergo ultrafast internal conversion (IC) allowing for an efficient radiationless decay towards lower-lying electronic states. ${ }^{7-15}$ The role of intersystem crossing (ISC) in the process of photostability is, however, much less discussed, 15 probably because it is thought to be a much slower process in comparison to $\mathrm{IC}^{20}$ and also because the quantum yields of triplet states population in DNA and RNA nucleobases are generally very small and thus difficult to access from the experimental point of view. $10 \mid 15 / 21$ We note, however, that ultrafast time scales for ISC in other organic molecules have been experimentally reported or predicted before. $22 \sqrt{36}$

In this work we present the first excited state dynamical study of a DNA nucleobase including singlet and triplet states. Such simulations are done using the newly developed surfacehopping method SHARC. 37 SHARC stands for surface Hopping including ARbitrary Couplings. With SHARC one can treat non-adiabatic and spin-orbit couplings (which mediate IC and ISC, respectively) on equal footing. The applicability of SHARC to include spin-orbit as well as dipole couplings is documented in Refs. $\frac{37+39}{39}$ Here, SHARC is employed to investigate the role of the triplet states in the deactivation of cytosine within the framework of nonadiabatic molecular dynamics based on ab initio multiconfigurational methods. Such study is necessary to provide a mechanistic insight that goes beyond what can be learned from quantum chemical calculations alone.

Cytosine presents three tautomers: enol-, keto- and keto-imine-cytosine. Keto-cytosine is the biological relevant tautomer found in the DNA's nucleotides linked to the deoxyribose sugar moiety and the only one for which a crystalline structure exists. $\underline{40}$ Therefore, here we focus on keto-cytosine. Several stationary points ${ }^{3 / 41 / 42}$ of keto-cytosine, including two43,48 and three-state 49 conical intersections involved in the process of IC have been calculated with ab initio methods. Time-dependent calculations have indicated that the dynamical behavior of cytosine after photoexcitation is one of the most complicated among nucleobases, involving delocalization of the excited wave packet and relaxation through multiple competing pathways in the singlet excited state manifold. 652 54 The possible triplet state formation via ISC along the internal conversion pathway of excited singlet keto-cytosine has been discussed by Merchán et al. ${ }^{55 \mid 56}$ in the light of quantum chemical calculations.

Our ab initio molecular dynamic simulations are performed on seven states simultaneously: four singlets and three triplets. Energies, energy gradients, non-adiabatic and spinorbit couplings are computed on-the-fly using the state-average Complete Active Space SelfConsistent Field (CASSCF) method. ${ }^{[5 / 58}$ Further details are given in the Supporting Information (SI). The first singlet excited state, $\mathrm{S}_{1}$, has $\pi \pi^{*}$ character at the equilibrium geometry and it is bright while the states higher in energy, $\mathrm{S}_{2}$ and $\mathrm{S}_{3}$, correspond to dark $n \pi^{*}$ excitations, i.e. they have negligible oscillator strengths when vertically excited. The order of states at equilibrium geometry is not altered when going to higher levels of theory that include dynamical correlation (see Table S2 of SI). However, the on-the-fly approach used in this work prohibits the use of higher level methods such as CASPT2 and therefore we employ CASSCF. The Franck-Condon region -from which excitations take place- does not only comprise the equilibrium geometry but also slightly distorted geometries. These distortions are due to the different vibrations included within the zero- 
point energy of the system. Because in cytosine rather small deviations of the equilibrium geometry lead to a different ordering of the state character, the $\mathrm{S}_{2}$ and $\mathrm{S}_{3}$ states can also be bright states and contribute to the absorption spectrum (see Refs. $\frac{6153}{}$ and Fig. S1 of SI). The character of the lowest three excited triplet states at equilibrium is $\pi \pi^{*}$ for $\mathrm{T}_{1}$ and $\mathrm{T}_{2}$, and $n \pi^{*}$ for $\mathrm{T}_{3}$. A comprehensive report of vertical excitation energies at different levels of theory can be found in the SI.

In order to obtain a global picture of the relaxation mechanisms of keto-cytosine, we have first used initial conditions spanning the whole first absorbtion band of the UV spectrum, i.e. covering excitation energies from ca 4 to $7 \mathrm{eV}$. As explained above, this requires launching trajectories from the first three excited states, $S_{1}$, $\mathrm{S}_{2}$ and $\mathrm{S}_{3}$. Most time-resolved spectroscopic experiments in cytosine $\mathrm{e}^{912 / 48 / 59 \mid 60}$ use a pumping wavelength of $267 \mathrm{~nm}(4.64 \mathrm{eV})$, just below the center of the first absorption band located at $260 \mathrm{~nm}(4.77 \mathrm{eV})$. In order to narrow the initial conditions to the energy range corresponding to the experimental one, we have also analyzed the results (Figure S2 of SI) limited to the bandwidth $4.75 \pm 0.25 \mathrm{eV}$, just below our theoretically predicted first absorption band maximum. Also in this energy range, states $S_{1}$ to $\mathrm{S}_{3}$ are excited. The results from both energy ranges qualitatively agree with each other so that we will limit the discussion to the more general broad range.

Figure 1 displays the time evolution of all the state populations and Fig. 2 summarizes the most important deactivation paths found in keto-cytosine with SHARC, including decay times and branching efficiencies. One should note that because the calculations are done at levels of theory which do not include sufficient dynamic correlation, the potential energy surface for the dynamics is not accurate enough to derive quantitative conclusions. Since the energy gaps between the states are highly dependent on the level of theory, the branching efficiencies and the decay times should be considered as an informative basis rather than quantitative numbers. We indicate the total branching efficiencies over the whole simulation time so that in some cases numbers can be higher than the initial population. Percentages not adding to $100 \%$ are due to minor pathways not indicated. The decay times are obtained fitting the net amount of hops between two particular states (see Fig. S3 and Table S1 of SI) to an exponential function. The branching efficiencies given in $\%$ are also graphically indicated by arrows of different thickness according to their importance. After photo-excitation, which corresponds to time zero in our simulations, the population of the $\pi \pi^{*}$ is distributed as $13 \%$ in $\mathrm{S}_{3}, 47 \%$ in $\mathrm{S}_{2}$, and $40 \%$ in $\mathrm{S}_{1}$, as dictated by the weight of the oscillator strength of each state. Since the character of a state can adiabatically change during the simulation, hereafter we shall refer to the states by its energetic order rather than by their character.

Analyzing the $13 \%$ population of $\mathrm{S}_{3}, 10 \%$ relaxes non-adiabatically to the $\mathrm{S}_{2}$ and from there to the $\mathrm{S}_{1}$ within $25 \mathrm{fs}$. After ca $155 \mathrm{fs}$, the system populates the electronic ground state $\mathrm{S}_{0}$. The remaining $3 \%$ of the population of $\mathrm{S}_{3}$ deactivates directly to $\mathrm{S}_{1}$ via a threefold degeneracy $\mathrm{S}_{3} / \mathrm{S}_{2} / \mathrm{S}_{1}$, as proposed in Ref. ${ }^{51}$ This process is calculated to be slower than the previous one, with a time constant of 110 fs. Most of the population in $\mathrm{S}_{2}$ transfers preferably to the lowerlying electronic states within less than 100 fs. Also in this case the process of IC is possible via a cascade of subsequent $\mathrm{S}_{2} / \mathrm{S}_{1}$ and $\mathrm{S}_{1} / \mathrm{S}_{0}$ conical intersections, or directly via three-state conical intersections $\mathrm{S}_{2} / \mathrm{S}_{1} / \mathrm{S}_{0}$, as proposed in Refs. Both pathways to the ground state are relevant, in agreement with the time-dependent simulations of Ref. 53 As deduced from the time constants, the three-state conical intersection pathway is faster ( $25 \mathrm{fs}$ ) than the two-step pathway (25 fs / 155 fs). As noticeable from Fig. 1, the first encounter with a conical intersection takes place within only $10 \mathrm{fs}$; however, complete depopulation of the $S_{2}$ state takes roughly 400 fs. The $\mathrm{S}_{1}$ state starts with $40 \%$ of population. Loss of population is possible via IC to the $\mathrm{S}_{0}$ state but due to gains from $\mathrm{S}_{2}$ and $\mathrm{S}_{3}$ the net effect (see Fig. 1) is that ca $30 \%$ of population remains for ca $100 \mathrm{fs}$, before decaying exponentially to zero in about 0.5 ps.

Conspicuously, due to near degeneracies be- 


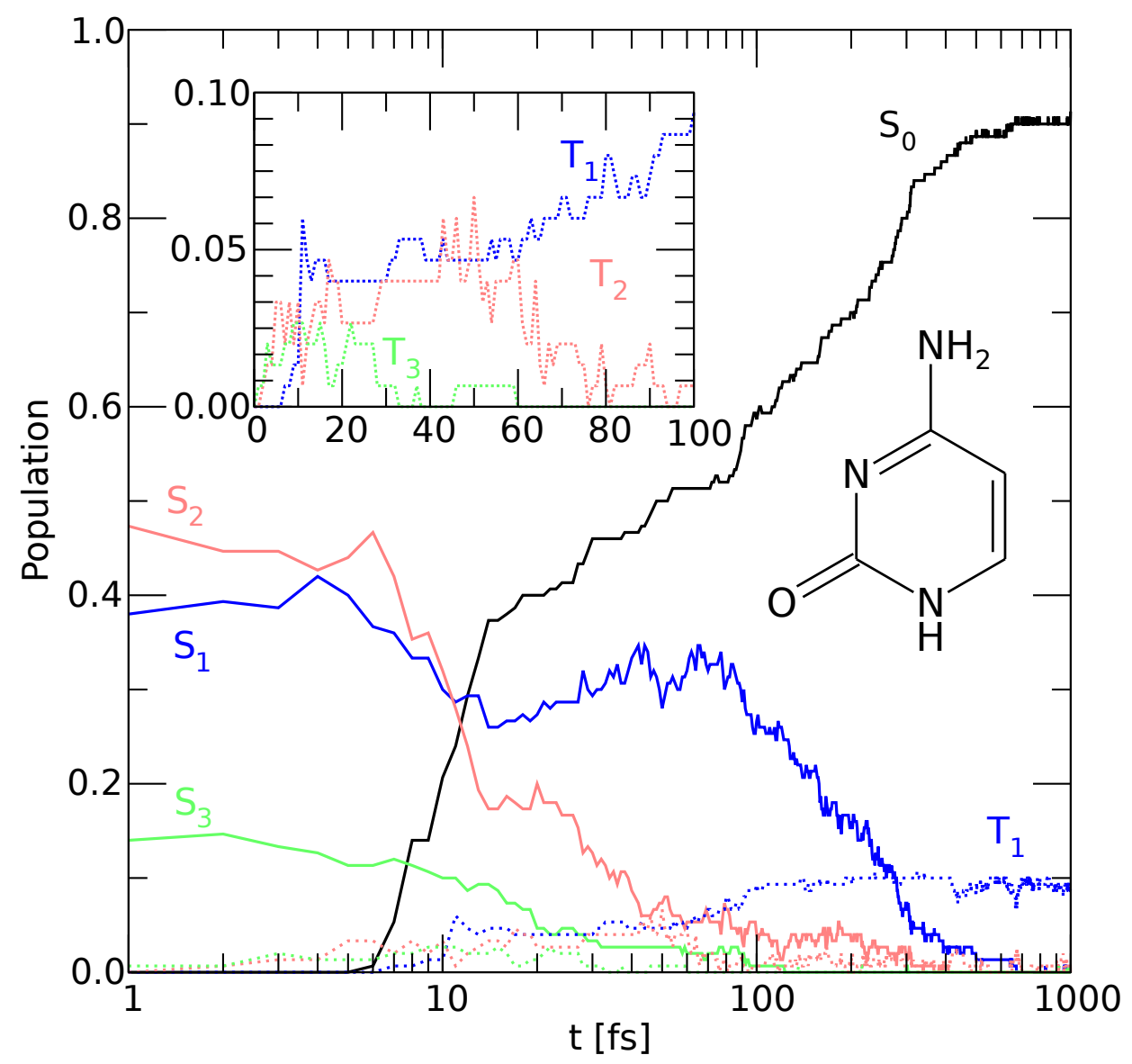

Figure 1: Time evolution of the singlet (solid) and triplet (dotted) states during the first picosecond. The inset zooms the first $100 \mathrm{fs}$. The $\mathrm{S}_{0}$ ground state population is in black, $\mathrm{S}_{1} / \mathrm{T}_{1}$ states populations are in red, $\mathrm{S}_{2} / \mathrm{T}_{2}$ in blue and $\mathrm{S}_{3} / \mathrm{T}_{3}$ in green. 


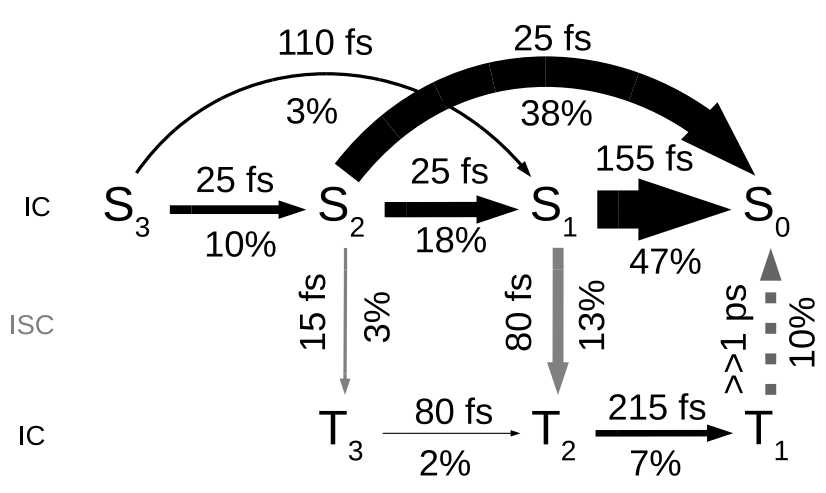

Figure 2: Deactivation pathways of ketocytosine including internal conversion (IC, in black) and intersystem crossing (ISC, in grey). The propensity of each path is sketched by the thickness of the arrows. The dotted line indicates the deactivation pathway of $T_{1}$.

tween singlet and triplet states, the trajectories clearly show that besides IC, ISC also takes place during the first tens of fs. Triplet states are populated strikingly fast (see inset in Fig. 1). ISC mainly occurs from $S_{1}$ to $T_{2}$ but in a lesser extent from $\mathrm{S}_{2}$ to $\mathrm{T}_{3}$, see Fig. 2 and Figure S3b in SI. In turn, the trajectories in the $\mathrm{T}_{3}$ state relax via $\mathrm{IC}$ to the $\mathrm{T}_{2}$ state and those in $\mathrm{T}_{2}$ quickly convert to $\mathrm{T}_{1}$. A minority of the trajectories deactivate directly from $T_{3}$ to $T_{1}$ via a threefold degeneracy. The simulations show that during the first 20 fs there is a degeneracy of many states $\left(\mathrm{S}_{0}, \mathrm{~S}_{1}, \mathrm{~S}_{2}, \mathrm{~T}_{1}\right.$ and $\left.\mathrm{T}_{2}\right)$, allowing for efficient population transfer. At $\mathrm{t}=20$ fs, $\mathrm{T}_{1}$ and $\mathrm{T}_{2}$ are populated by ca $5 \%$ and $\mathrm{T}_{3}$ by half of it. The $T_{3}$ state is depopulated in less than 100 fs. Most of the population of $T_{2}$ has radiationless decayed to $T_{1}$ within 200 fs but since it is constantly replenished from $\mathrm{S}_{1}$, a small amount of population persists until 1 ps. The trajectories show that as a consequence of the ISC process and the subsequent IC between triplet states, the $\mathrm{T}_{1}$ state has gained significant population (ca 10\%) after 140 fs. This population is stable after the propagation time (1 ps) and most likely can survive during several ps or even ns,,$\frac{60}{6}$ contributing to long-lived transients. The $10 \%$ of population trapped in the lowest triplet state agrees with the yield of the dark state $(9 \pm 7 \%)$, from which ISC can take place, measured by Hare et al. 15

In general, most of the time decay constants calculated are below 100 fs. Ultrafast timeresolved fs pump-probe experiments ${ }^{7 / 12|59| 61}$ for cytosine (and other nucleobases) provide transients with three time scales: one in the fs time regime, $\tau_{1}<100 \mathrm{fs}$, and two slower ones, in the ps scale, $\tau_{2}$ and $\tau_{3}$-the latter one even in the ns regime. ${ }^{60}$ As recently noted in Refs., ${ }^{42 / 48 / 62}$ experiments involving ionization integrate over different photoelectron energies or ionic fragments. Interestingly, it has been also demonstrated ${ }^{48}$ that different molecular fragments show different time scales (corresponding to very different relaxation pathways to the ground state). Therefore, the three time constants mentioned above correspond most likely to an average over the many deactivation routes taking place in the nucleobases. As such, our calculated time scales for each of the multitude of relaxation pathways can only contribute to the averaged $\tau_{1}$ decay time measured in Refs. ${ }^{59 / 60}$ Sub-picosecond ISC occurring before vibrational relaxation in the $\mathrm{S}_{1}$ state has been also proposed in DNA base analogues (4-thiothymidine and 6-thioguanosine) 22 24/63 lending further support to the participation of triplet states in the relaxation process of cytosine. The longest decay time $\tau_{3}$ can be associated with the ISC from $\mathrm{T}_{1}$ to the ground state $\mathrm{S}_{0}$. Unfortunately, it is difficult to speculate about the origin of the intermediate $\tau_{2}$ since none of our obtained time scales (recall Figure 2) are in the ps time scale.

Particularly interesting is to note that the obtained ISC mechanism $\left(\mathrm{S}_{1} \rightarrow \mathrm{T}_{2} \rightarrow \mathrm{T}_{1}\right)$ is different from the one previously proposed in the literature, based solely on quantum chemistry. The calculations of Merchán and coworkers ${ }^{55}$ propose $\mathrm{S}_{1} \rightarrow \mathrm{T}_{1}$ transitions. Their calculations find a minimum in the $S_{1}$ potential from where the $S_{1}$ and $T_{1}$ states are almost degenerate along the minimum energy path. A substantial spin-orbit coupling of $20-30 \mathrm{~cm}^{-1}$ is found along this path. $[55$ In their calculations, the $\mathrm{S}_{1} / \mathrm{T}_{1}$ crossing corresponds to a transition between the ${ }^{1} \pi \pi^{*}$ and the ${ }^{3} n \pi^{*}$ states, in agreement with the El-Sayed selection rules for ISC.6465 In contrast, in our calculations, 
the ISC takes place fundamentally between the ${ }^{1} n \pi^{*}\left(\mathrm{~S}_{1}\right)$ and the ${ }^{3} n \pi^{*}\left(\mathrm{~T}_{2}\right)$ states, while the ${ }^{3} \pi \pi^{*}$ state is the $\mathrm{T}_{1}$. Accordingly, and in agreement with the El-Sayed rules, the spin-orbit coupling between $\mathrm{S}_{1}$ and $\mathrm{T}_{1}$ is larger (ca 15$20 \mathrm{~cm}^{-1}$ in average, maximum $40 \mathrm{~cm}^{-1}$ ) than between the $\mathrm{S}_{1}$ and $\mathrm{T}_{2}$ state (ca $5 \mathrm{~cm}^{-1}$ in average). However, the $\mathrm{S}_{1} \rightarrow \mathrm{T}_{2}$ transition is predominant because the $\mathrm{T}_{2}$ state is separated from the $S_{1}$ by a very small energy gap $(<0.05 \mathrm{eV})$ for many geometries along the key reaction path. In contrast, the energy gap between the $S_{1}$ and $T_{1}$ states is at least $0.1 \mathrm{eV}$ for most of the geometries on the same path and hence hopping is not efficient. Clearly, the close degeneracy between the states of different multiplicity can compensate for very small spinorbit coupling and drive fast ISC, as recently discussed by Worth and coworkers $\frac{3435}{3 n}$ in benzene (see also Ref. .66 ). Thus, the $\mathrm{S}_{1} \rightarrow \mathrm{T}_{2}$ transition dominates the ISC process. This result illustrates the importance of time-dependent dynamical simulations over quantum chemistry calculations alone. In view of our simulations, the $\mathrm{S}_{1}$ can be considered the precursor state from which the largest ISC takes place before vibrational cooling occurs - as proposed in Ref. ${ }^{15}$ or in Refs. 16$] 17$ for uracil and thymine, respectively.

Another important mechanistic conclusion obtained from the present simulations is that ISC coming from higher states also contributes to the deactivation of keto-cytosine. Common belief is that only spin-flips from the low-lying $\mathrm{S}_{1}$ state are relevant because ISC transitions in organic molecules without heavy elements are not considered efficient in the fs timescale. Contrary to that, $\mathrm{S}_{2} \rightarrow \mathrm{T}_{3}$ transitions are possible in keto-cytosine, leading to population transfer of ca $3 \%$ after few fs. ISC channels from singlet states higher than the $\mathrm{S}_{1}$ have been also suggested in e.g. 4-thiothymidine by Reichardt et al. ${ }^{\sqrt{22}}$ or in uracil and thymine by Etinski et al. 19

Further insight into the different competing pathways can be obtained by characterizing the molecular geometries that allow for an efficient population transfer. Figure 3 collects snapshots of archetypal geometries where two or three states are found (near) degenerate (energy less
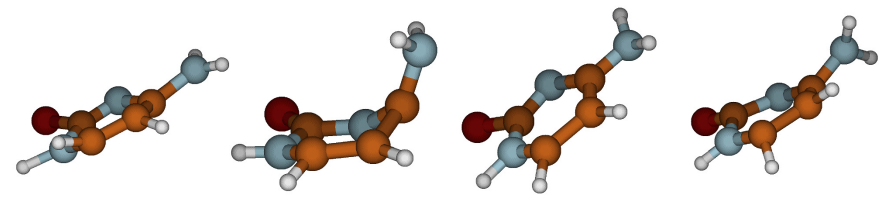

(a) $\mathrm{S}_{0} / \mathrm{S}_{1}$

(b) $S_{0} / S_{1}$

(c) $\mathrm{S}_{0} / \mathrm{S}_{1} / \mathrm{S}_{2}$

(d) $S_{1} / S_{2} / S_{3}$

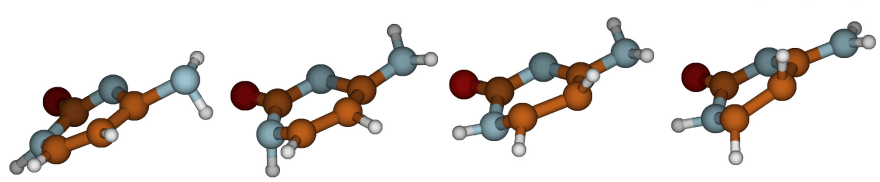

$\begin{array}{ll}\text { (e) } S_{1} / T_{2} & \text { (f) } S_{1} / T_{2}\end{array}$

(q) $S_{1} / T_{2}$

(h) $\mathrm{S}_{2} / \mathrm{T}_{3}$
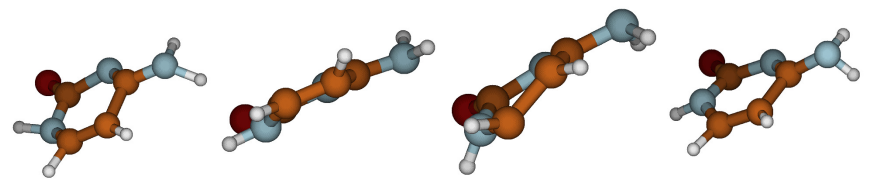

(i) $T_{1} / T_{2}$

(i) $T_{1} / T_{2}$

(k) $T_{2} / T_{3}$

(I) $T_{1} / T_{2} / T_{3}$

Figure 3: Characteristic geometries displaying near degeneracies between two or three states.

than $0.1 \mathrm{eV})$. Among the geometries where the $\mathrm{S}_{0}$ and $\mathrm{S}_{1}$ states are close in energy, two geometries similar to the twist and sofa $\mathrm{S}_{0} / \mathrm{S}_{1}$ conical intersections reported by Kotur et al. $\stackrel{48}{ }$ could be identified. Figure 3a shows our twist structure, with the carbon atoms twisted by about $70^{\circ}$ around the $\mathrm{C}=\mathrm{C}$ double bond. The sofa conical intersection, Fig. 3b, is characterized by the $\mathrm{NH}_{2}$ nitrogen displaced from the plane, giving the ring structure the appearance of a sofa. A 3-state near degeneracy between the $\mathrm{S}_{0}, \mathrm{~S}_{1}$ and $\mathrm{S}_{2}$ states is a frequent event in ketocytosine, see Fig. 3c. With the $\mathrm{C}=\mathrm{O}$ bond elongated to $1.42 \AA$, the $\mathrm{C}=\mathrm{C}$ bond between the two $\mathrm{CH}$ groups elongated to $1.52 \AA$, and the $\mathrm{NH}_{2}$ group pyramidalized to an angle of $45^{\circ}$ at the nitrogen, this geometry resembles the structure first optimized by Blancafort et al. $\frac{49}{4}$ and later on by Kistler and coworkers. $\frac{51}{51}$ Interestingly, this structure is also similar to the semiplanar $\mathrm{S}_{0} / \mathrm{S}_{1}$ conical intersection published by Barbatti et al. 54

Figure 3d shows a 3-state degeneracy involving the $\mathrm{S}_{1}, \mathrm{~S}_{2}$ and $\mathrm{S}_{3}$ states. Here, the $\mathrm{C}=\mathrm{C}$ bond is elongated to $1.54 \AA$ and the carbon atoms are twisted about $56^{\circ}$ around the $\mathrm{C}=\mathrm{C}$ double bond. The dihedral at the $\mathrm{NH}_{2}$ nitrogen is only $22^{\circ}$ and therefore, the $\mathrm{NH}_{2}$ group is rotated by about $35^{\circ}$ around the $\mathrm{N}-\mathrm{C}$ bond rather than pyramidalized. This geometry is differ- 
ent from the structures proposed by Kistler et al. $\underline{51}$ The trajectories show that singlet and triplet states are usually near degenerate if the $\mathrm{NH}_{2}$ group is pyramidalized and starts rotating around the $\mathrm{C}-\mathrm{N}$ bond, as in Fig. 3e, and/or if the hydrogen of the $\mathrm{NH}$ group in para position shows strong out-of-plane oscillations, as in Fig. 3f. Also, when the other hydrogens of the ring show strong out-of-plane movement, near singlet/triplet degeneracies take place, see Figs. 3g,h. To the best of our knowledge no conical intersections between triplet states are reported so far -most likely because only $\mathrm{T}_{1}$ was believed to be involved in ISC. Figs. 3i,j,k show geometries where two triplet states are degenerate. A degeneracy between $\mathrm{T}_{1}$ and $\mathrm{T}_{2}$ (Figs. 3i) occurs when the $\mathrm{C}-\mathrm{C}$ bond next to the $\mathrm{NH}_{2}$ group is elongated up to $1.68 \AA$. Additionally, the $\mathrm{NH}_{2}$ group is pyramidalised and the hydrogen of the $\mathrm{NH}$ group in para position shows out-of-plane movement. Other structures like the one given in Fig. $3 \mathrm{j}$ show a twist of the $\mathrm{C}=\mathrm{C}$ double bond and a slight out-of-plane movement of the whole $\mathrm{NH}$ group, leading to a degeneracy of the $T_{1}$ and $T_{2}$ states. The geometries where $T_{2}$ and $T_{3}$ come close in energy show similar features: pyramidalisation of the $\mathrm{NH}_{2}$ group and the out-of-plane movements of the ring hydrogens, see Fig. 3k. Additionally, the $\mathrm{C}=\mathrm{C}$ double bond is twisted and rotated out-of-plane. Additionally, triple degeneracies of triplet states have been also identified, see Fig. 3l, although they are not operative within 1 ps. Here, the $\mathrm{NH}_{2}$ group is pyramidalized by $67^{\circ}$ and the $\mathrm{C}-\mathrm{C}$ bond next to the $\mathrm{NH}_{2}$ group is elongated to $1.71 \AA$. This indicates that IC from $\mathrm{T}_{3}$ is not only possible via subsequent $\mathrm{T}_{3} / \mathrm{T}_{2}$ and $\mathrm{T}_{2} / \mathrm{T}_{1}$ conical intersections, but also directly from $\mathrm{T}_{3} \rightarrow \mathrm{T}_{1}$.

In summary, our work provides a theoretical rationale for the nuclear factors that make ISC compete with IC in isolated keto-cytosine. As this study demonstrates, the fate of energy relaxation in many complex molecules cannot be revealed by a frozen picture, i.e. quantum chemical calculations at optimized geometries. Instead, a time-dependent picture is necessary, where both IC and ISC compete as dynamical events. ISC is generally quoted in textbooks ${ }^{20}$ as a slower process in comparison to IC. As a result, many scientists are unaware that ISC can be also an ultrafast process, not only when heavy atoms are present (see e.g.67), but also in organic molecules -as this study evidences.

Acknowledgement This work is financed by the Deutsche Forschungsgemeinschaft (DFG) within the project GO $1059 / 6-1$, by the German Federal Ministry of Education and Research within the research initiative PhoNa, the Dirección General de Investigación of Spain under Project No. CTQ2008-06760, a Juan de la Cierva contract, and the European COST Action CM0702. We thank T. Weinacht for helpful discussions regarding cytosine experiments. Generous allocation of computer time in Jena (Computer Center) and Vienna (Vienna Scientific Cluster) is gratefully acknowledged.

\section{Supporting Information Avail- able}

Computational details, calculated UV absorption spectra as well as additional results.

\section{References}

(1) Nylund, R.; Leszczynski, D. Mobile Phone Radiation Causes Changes in Gene and Protein Expression in Human Endothelial Cell Lines and the Response Seems to be Genome- and Proteome-Dependent. Proteomics 2006, 6, 4769-4780.

(2) Crespo-Hernández, C. E.; Cohen, B.; Kohler, B. Base Stacking Controls Excited-State Dynamics in A-T DNA. Nature 2005, 436, 1141-1144.

(3) Shukla, M. K.; Leszczynski, J. Radiation Induced Molecular Phenomena in Nucleic Acids. 2008 (Springer).

(4) Schultz, T.; Samoylova, E.; Radloff, W.; Hertel, V. I.; Sobolewski, A. L.; Domcke, W. Efficient Deactivation of a Model Base Pair Via Excited-State Hydrogen Transfer. Science 2004, 306, 1765-1768. 
(5) Abo-Riziq, A.; Grace, L.; Nir, E.; Kabelac, M.; Hobza, P.; Vries, M. S. d. Photochemical Selectivity in GuanineCytosine Base-Pair Structures. Proc. Natl. Acad. Sci. USA 2005, 102, 20-23.

(6) Barbatti, M.; Aquino, A. J. A.; Szymczak J. J.; Nachtigallová, D.; Hobza, P.; Lischka, H. Relaxation Mechanisms of UV-PhotoexcitedDNA and RNA Nucleobases. P. Natl. Acad. Sci. USA 2010, 107, 21453-21458.

(7) Peon, J.; Zewail, A. H. DNA/RNA Nucleotides and Nucleosides: Direct Measurement of Excited-State Lifetimes by Femtosecond Fluorescence Up-Conversion. Chem. Phys. Lett. 2001, 348, 255-262.

(8) Pecourt, J. M. L.; Peon, J.; Kohler, B. Ultrafast Internal Conversion of Electronically Excited RNA and DNA Nucleosides in Water. J. Am. Chem. Soc. 2000, 122, 9348-9349.

(9) Kang, H.; Lee, K. T.; Jung, B.; Ko, Y. J.; Kim, S. K. Intrinsic Lifetimes of the Excited State of DNA and RNA Bases. $J$. Am. Chem. Soc. 2002, 124, 12958-12959.

(10) Crespo-Hernández, C. E.; Cohen, B.; Hare, P. M.; Kohler, B. Ultrafast ExcitedState Dynamics in Nucleic Acids. Chem. Rev. 2004, 104, 1977-2020.

(11) Ullrich, S.; Schultz, T.; Zgierski, M. Z.; Stolow, A. Electronic Relaxation Dynamics in DNA and RNA Bases Studied by Time-Resolved Photoelectron Spectroscopy. Phys. Chem. Chem. Phys. 2004, 6, 2796-2801.

(12) Canuel, C.; Mons, M.; Piuzzi, F.; Tardivel, B.; Dimicoli, I.; Elhanine, M. Excited States Dynamics of DNA and RNA Bases: Characterization of a Stepwise Deactivation Pathway in the Gas Phase. J. Chem. Phys. 2005, 122, 074316.
(13) Kwok, W. M.; Ma, C.; Phillips, D. L. Femtosecond Time- and WavelengthResolved Fluorescence and Absorption Spectroscopic Study of the Excited States of Adenosine and an Adenine Oligomer. $J$. Am. Chem. Soc. 2006, 128, 11894-11905.

(14) Vayá, I.; Gustavsson, T.; Miannay, F. A.; Douki, T.; Markovitsi, D. Fluorescence of Natural DNA: From the Femtosecond to the Nanosecond Time Scales. J. Am. Chem. Soc. 2010, 132, 11834-11835.

(15) Hare, P. M.; Crespo-Hernández, C. E.; Kohler, B. Internal Conversion to the Electronic Ground State Occurs via Two Distinct Pathways for Pyrimidine Bases in Aqueous Solution. P. Natl. Acad. Sci. USA 2007, 104, 435-440.

(16) Hare, P. M.; Crespo-Hernández, C. E.; Kohler, B. Solvent-Dependent Photophysics of 1-Cyclohexyluracil: Ultrafast Branching in the Initial Bright State Leads Nonradiatively to the Electronic Ground State and a Long-Lived ${ }^{1} \mathrm{n} \pi^{*}$ State. J. Phys. Chem. B 2006, 110, 18641-18650.

(17) Hare, P. M.; Middleton, C. T.; Mertel, K. I.; Herbert, J. M.; Kohler, B. Time-Resolved Infrared Spectroscopy of the Lowest Triplet State of Thymine and Thymidine. Chem. Phys. 2008, 347, 383 $-392$.

(18) Kwok, W. M.; Ma, C.; Phillips, D. L. A Doorway State Leads to Photostability or Triplet Photodamage in Thymine DNA. J. Am. Chem. Soc. 2008, 130, 5131-5139.

(19) Etinski, M.; Fleig, T.; Marian, C. M. Intersystem Crossing and Characterization of Dark States in the Pyrimidine Nucleobases Uracil, Thymine, and 1Methylthymine. J. Phys. Chem. A 2009, 113, $11809-11816$.

(20) McQuarrie, D. A.; Simon, J. D. Physical Chemistry 1997 (University Science Books). 
(21) Cadet, J.; Vigny, P. The Photochemistry of Nucleic Acids. Bioorganic Photochemistry 1990, 1, 1-272.

(22) Reichardt, C.; Crespo-Hernández, C. E. Room-Temperature Phosphorescence of the DNA Monomer Analogue 4Thiothymidine in Aqueous Solutions after UVA Excitation. J. Phys. Chem. Lett. 2010, 1, $2239-2243$.

(23) Reichardt, C.; Guo, C.; CrespoHernández, C. E. Excited-State Dynamics in 6-Thioguanosine from the Femtosecond to Microsecond Time Scale. J. Phys. Chem. B 2011, 115, 3263 - 3270.

(24) Reichardt, C.; Crespo-Hernández, C. E. Ultrafast Spin Crossover in 4Thiothymidine in an Ionic Liquid. Chem. Commun. 2010, 46, 5963 - 5965.

(25) Cavaleri, J. J.; Prater, K.; Bowman, R. M. An Investigation of the Solvent Dependence on the Ultrafast Intersystem Crossing Kinetics of Xanthone. Chem. Phys. Lett. 1996, 259, 495 - 502.

(26) Tamai, N.; Asahi, T.; Masuhara, H. Intersystem Crossing of Benzophenone by Femtosecond Transient Grating Spectroscopy. Chem. Phys. Lett. 1992, 198, 413 - 418.

(27) Aloise, S.; et al. The Benzophenone $\mathrm{S}_{1}\left(\mathrm{n}, \pi^{*}\right) \rightarrow \mathrm{T}_{1}\left(\mathrm{n}, \pi^{*}\right)$ States Intersystem Crossing Reinvestigated by Ultrafast Absorption Spectroscopy and Multivariate Curve Resolution. J. Phys. Chem. A 2008, 112, 224-231.

(28) Zugazagoitia, J. S.; Collado-Fregoso, E.; Plaza-Medina, E. F.; Peon, J. Relaxation in the Triplet Manifold of 1Nitronaphthalene Observed by Transient Absorption Spectroscopy. J. Phys. Chem. A 2009, 113, 805-810.

(29) Ghosh, R.; Palit, D. K. Ultrafast Dynamics of the Excited States of 1-(p-Nitrophenyl)-2(Hydroxymethyl)Pyrrolidine. J. Phys. Chem. A 2012, 116, 1993-2005.
(30) Minns, R. S.; Parker, D. S. N.; Penfold, T. J.; Worth, G. A.; Fielding, H. H. Competing Ultrafast Intersystem Crossing and Internal Conversion in the Channel 3 Region of Benzene. Phys. Chem. Chem. Phys. 2010, 12, 15607-15615.

(31) Crespo-Hernández, C. E.; Burdzinski, G.; Arce, R. Environmental Photochemistry of Nitro-PAHs: Direct Observation of Ultrafast Intersystem Crossing in 1Nitropyrene. J. Phys. Chem. A 2008, 112, 6313-6319.

(32) Reichardt, C.; Vogt, R. A.; CrespoHernández, C. E. On the Origin of Ultrafast Nonradiative Transitions in Nitro-Polycyclic Aromatic Hydrocarbons: Excited-State Dynamics in 1-Nitronaphthalene. J. Chem. Phys. 2009, 131, 224518

(33) Yang, C.; Su, H.; Sun, X.; George, M. W. Ultrafast Formation of the Benzoic Acid Triplet upon Ultraviolet Photolysis and its Sequential Photodissociation in Solution. J. Chem. Phys. 2012, 136, 204507

(34) Parker, D. S. N.; Minns, R. S.; Penfold, T. J.; Worth, G. A.; Fielding, H. H. Ultrafast Dynamics of the $\mathrm{S}_{1}$ Excited State of Benzene. Chem. Phys. Lett. 2009, 469, $43-47$.

(35) Penfold, T. J.; Worth, G. A. The Effect of Molecular Distortions on Spin-Orbit Coupling in Simple Hydrocarbons. Chem. Phys. 2010, 375, $58-66$.

(36) Etinski, M.; Tatchen, J.; Marian, C. M. Time-Dependent Approaches for the Calculation of Intersystem Crossing Rates. J. Chem. Phys. 2012, 134, 154105.

(37) Richter, M.; Marquetand, P.; GonzáezVáquez, J.; Sola, I.; González, L. SHARC: Ab Initio Molecular Dynamics with Surface Hopping in the Adiabatic Representation Including Arbitrary Couplings. J. Chem. Theory Comput. 2011, 7, 12531258. 
(38) Marquetand, P.; Richter, M.; GonzálezVázquez, J.; Sola, I.; González, L. Nonadiabatic Ab Initio Molecular Dynamics Including Spin-Orbit Coupling and Laser Fields. Faraday Discuss. 2011, 153, 261273.

(39) Bajo, J. J.; González-Vázquez, J.; Sola, I. R.; Santamaria, J.; Richter, M.; Marquetand, P.; González, L. Mixed QuantumClassical Dynamics in the Adiabatic Representation to Simulate Molecules Driven by Strong Laser Pulses. J. Phys. Chem. A 2012, 116, 2800-2807.

(40) Barker, D. L.; Marsh, R. E. The Crystal Structure of Cytosine. Acta Crystallogr. 1964, 17, 1581-1587.

(41) Blancafort, L.; Bearpark, M. J.; Robb, M. A. Computational Modeling of Cytosine Photophysics and Photochemistry: From the Gas Phase to DNA. In Shukla, M. K.; Leszczynski, J. eds. Radiation Induced Molecular Phenomena in Nucleic Acids (Springer Netherlands), vol. 5 of Challenges and Advances in Computational Chemistry and Physics 2008 pp. 473-492.

(42) Matsika, S.; Zhou, C.; Kotur, M.; Weinacht, T. C. Combining Dissociative Ionization Pump-Probe Spectroscopy and $\mathrm{Ab}$ Initio Calculations to Interpret Dynamics and Control Through Conical Intersections. Faraday. Discuss. 2011, 153, 247-260.

(43) Ismail, N.; Blancafort, L.; Olivucci, M.; Kohler, B.; Robb, M. A. Ultrafast Decay of Electronically Excited Singlet Cytosine via a $\pi, \pi^{*}$ to $n_{o}, \pi^{*}$ State Switch. J. Am. Chem. Soc. 2002, 124, 6818-6819.

(44) Merchán, M.; Serrano-Andrés, L. Ultrafast Internal Conversion of Excited Cytosine via the Lowest $\pi \pi^{*}$ Electronic Singlet State. J. Am. Chem. Soc. 2003, 125, 8108-8109.

(45) Tomić, K.; Tatchen, J.; Marian, C. M. Quantum Chemical Investigation of the
Electronic Spectra of the Keto, Enol, and Keto-Imine Tautomers of Cytosine. J. Phys. Chem. A 2005, 109, 8410-8418.

(46) Blancafort, L.; Bertran, J.; Sodupe, M. Triplet $\left(\pi, \pi^{*}\right)$ Reactivity of the GuanineCytosine DNA Base Pair: Benign Deactivation versus Double Tautomerization via Intermolecular Hydrogen Transfer. J. Am. Chem. Soc. 2004, 126, 12770-12771.

(47) Blancafort, L. Energetics of Cytosine Singlet Excited-State Decay Paths -a Difficult Case for CASSCF and CASPT2. Photochem. Photobiol. 2007, 83, 603-610.

(48) Kotur, M.; Weinacht, T. C.; Zhou, C.; Kistler, K. A.; Matsika, S. Distinguishing Between Relaxation Pathways by Combining Dissociative Ionization Pump Probe Spectroscopy and Ab Initio Calculations: A Case Study of Cytosine. J. Chem. Phys. 2011, 134, 184309.

(49) Blancafort, L.; Robb, M. A. Key Role of a Threefold State Crossing in the Ultrafast Decay of Electronically Excited Cytosine. J. Phys. Chem. A 2004, 108, 10609 10614.

(50) Kistler, K. A.; Matsika, S. Radiationless Decay Mechanism of Cytosine: An Ab Initio Study with Comparisons to the Fluorescent Analogue 5-Methyl-2Pyrimidinone. J. Phys. Chem. A 2007, 111, 2650-2661.

(51) Kistler, K. A.; Matsika, S. ThreeState Conical Intersections in Cytosine and Pyrimidinone Bases. J. Chem. Phys. 2008, 128, 215102.

(52) Hudock, H. R.; Martínez, T. J. ExcitedState Dynamics of Cytosine Reveal Multiple Intrinsic Subpicosecond Pathways. Chem. Phys. Chem. 2008, 9, 2486-2490.

(53) González-Vázquez, J.; González, L. A Time-Dependent Picture of the Ultrafast Deactivation of Keto-Cytosine Including Three-State Conical Intersections. Chem. Phys. Chem. 2010, 11, 3617-3624. 
(54) Barbatti, M.; Aquino, A. J. A.; Szymczak, J. J.; Nachtigallova, D.; Lischka, H. Photodynamical Simulations of Cytosine: Characterization of the Ultrafast Bi-Exponential UV Deactivation. Phys. Chem. Chem. Phys. 2011, 13, 6145-6155.

(55) Merchán, M.; Serrano-Andrés, L.; Robb, M. A.; Blancafort, L. Triplet-State Formation Along the Ultrafast Decay of Excited Singlet Cytosine. J. Am. Chem. Soc. 2005, 127, 1820-1825.

(56) González-Luque, R.; Climent, T.; González-Ramírez, I.; Merchán, M.; Serrano-Andrés, L. Singlet-Triplet States Interaction Regions in DNA/RNA Nucleobase Hypersurfaces. J. Chem. Theory. Comput. 2010, 6, 2103-2114.

(57) Werner, H. J.; Knowles, P. J. A Second Order Multiconfiguration SCF Procedure with Optimum Convergence. J. Chem. Phys. 1985, 82, 5053-5063.

(58) Knowles, P. J.; Werner, H. J. An Efficient Second-Order MC SCF Method for Long Configuration Expansions. Chem. Phys. Lett. 1985, 115, $259-267$.

(59) Kosma, K.; Schröter, C.; Samoylova, E.; Hertel, I. V.; Schultz, T. Excited-State Dynamics of Cytosine Tautomers. J. Am. Chem. Soc. 2009, 131, 16939-16943.

(60) Ho, J.-W.; Yen, H.-C.; Chou, W.-K.; Weng, C.-N.; Cheng, L.-H.; Shi, H.-Q.; Lai, S.-H.; Cheng, P.-Y. Disentangling Intrinsic Ultrafast Excited-State Dynamics of Cytosine Tautomers. J. Phys. Chem. A 2011, 115, 8406-8418.

(61) Pecourt, J. M. L.; Peon, J.; Kohler, B. DNA Excited-State Dynamics: Ultrafast Internal Conversion and Vibrational Cooling in a Series of Nucleosides. J. Am. Chem. Soc. 2001, 123, 10370-10378.

(62) Kotur, M.; Weinacht, T.; Zhou, C.; Matsika, S. Following Ultrafast Radiationless Relaxation Dynamics with Strong Field Dissociative Ionization: A Comparison
Between Adenine, Uracil, and Cytosine. IEEE J. Sel. Top. Quantum El. 2012, 18, $187-194$.

(63) Martínez-Fernández, L.; González, L.; Corral, I. An Ab Initio Mechanism for Efficient Population of Triplet States in Cytotoxic Sulfur Substituted DNA Bases: The Case of 6-Thioguanine. Chem. Commun. 2012, 48, 2134-2136.

(64) Lower, S. K.; El-Sayed, M. A. The Triplet State and Molecular Electronic Processes in Organic Molecules. Chem. Rev. 1966, 66, 199-241.

(65) Braslavsky, S. E. Glossary of Terms Used in Photochemistry, 3rd edition (IUPAC recommendations 2006). Pure Appl. Chem. 2007, 79, 293-465.

(66) Marian, C. M. Spin-Orbit Coupling and Intersystem Crossing in Molecules. WIREs Comput. Mol. Sci. 2012, 2, 187 $-203$.

(67) Chergui, M. On the Interplay Between Charge, Spin and Structural Dynamics in Transition Metal Complexes. Dalton Trans. 2012. 
Femtosecond Intersystem Crossing in the DNA Nucleobase Cytosine Supporting Information

\section{Computational details regarding dynamical simulations and initial conditions}

The simulation of the deactivation dynamics of keto-cytosine in gas phase was performed using the semiclassical SHARC method ${ }^{1}$ that calculates non-adiabatic molecular dynamics simulations using Tully's fewest switches criterion. ${ }^{[2]}$ The nuclei are treated as classical point charges that obey Newton's equations of motion. ${ }^{[3}$ To follow the trajectory of the nuclei, the Velocity-Verlet algorithm ${ }^{4}$ was applied with a time step of 0.5 fs. The energy gradients that serve as an input for the Velocity-Verlet algorithm were analytically calculated in the electronic part of the simulation. In contrast to the nuclei, the electrons are treated by means of ab initio quantum mechanics. Here, the electronic wave function is expanded into a linear combination of basis functions, that represent the contribution of the different calculated electronic states to the total wave function. The time evolution of the quantum amplitudes is followed using the 5 th order Butcher algorithm ${ }^{5}$ with a time step of $5 \cdot 10^{-6}$ fs. The resulting values were corrected for decoherence effects using the method of Granucci and Persico with a parameter of $\alpha=0.1$ hartree. .6

Initial conditions for the trajectories are generated with a Wigner harmonic distribution of 5000 uncorrelated geometries and velocities. For this purpose, the equilibrium geometry was optimized and normal modes were calculated with the TURBOMOLE package, ${ }^{78}$ the B3LYP hybrid functional ${ }^{9110}$ and the TZVP basis set. 11 For each of the initial conditions, a singlepoint calculation employing the Complete Active Space Self-Consistent-Field approach ${ }^{12113}$ averaged over four singlet states with the MOLPRO ${ }^{14}$ package and the $6-31 \mathrm{G}^{*}$ basis set was done (see Section III for further electronic structure details). All 5000 initial conditions and their calculated oscillator strengths and vertical excitations were used to create a UV absorption spectrum as described in Ref. ${ }^{15}$ The spectrum, see Fig. S1, is in good agreement with that previously published in Ref. 16

Using Newton- $\mathrm{X}^{[17118}$ it is possible to estimate the instantaneous probability to excite keto-cytosine at every geometry to a particular excited state. Applying these probabilities to the calculated 5000 initial conditions, we obtain 591 trajectories starting from the $\mathrm{S}_{1}, 685$ starting from the $\mathrm{S}_{2}$ and 192 starting from the $\mathrm{S}_{3}$ state. Since the molecular dynamics simulations converge with notably less than 1468 trajectories, only 150 trajectories have been propagated. This ensemble consisted of 60,70 and 20 trajectories, starting from the $S_{1}, S_{2}$ and $S_{3}$ respectively. The results were statistically evaluated for all trajectories and checked for convergence. Each trajectory was propagated during 1 ps using time steps of 0.5 fs.

Population transfer of the semiclassical trajectories between the different electronic states was calculated according to the Tully's fewest switches criterion ${ }^{[2}$ in the adiabatic representation, including non-adiabatic couplings as well as spin-orbit couplings. In order to avoid unphysical hops and for computational reasons, the hopping probabilities were only calculated for states that, after a hop, would lead to an increase of potential energy of $1 \mathrm{eV}$ or less. After a hop, the kinetic energy was adjusted in order to conserve the total energy of the system. Therefore, the velocities (i.e. the kinetic energies) of the atoms were scaled along their current direction in order to keep the total energy constant.

If a trajectory populated the $\mathrm{S}_{0}$ state for at least $20 \mathrm{fs}$ with a coefficient greater than 0.99 , it was stopped automatically and it was assumed that the population in the $S_{0}$ remains constant for the rest of the simulation.

\section{Deactivation pathways with an excitation bandwidth of $4.75 \pm 0.25$ eV}

Figure S2 displays the time evolution of all the state populations when limiting the excitation 


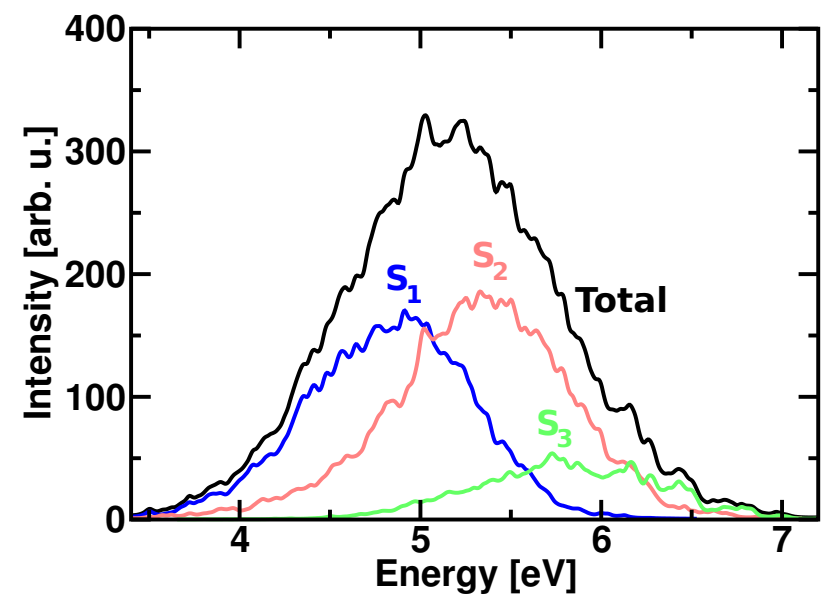

Figure S1: UV absorption spectrum of keto-cytosine.

bandwidth to $4.75 \pm 0.25 \mathrm{eV}$, in accord to the energy range corresponding to the experiments, see e.g. Ref. ${ }^{19}$ In this energy range only 42 trajectories are considered. As it can be seen the results are qualitatively the same as those shown in Fig. 1 of the manuscript using 150 trajectories in the broader energy range of 4 to 7 $\mathrm{eV}$. After 1000 fs the populations are converged to the same ratio, about $90 \%$ in $\mathrm{S}_{0}$ and $10 \%$ in $\mathrm{T}_{1}$. Moreover, the $\mathrm{S}_{0}$ gains about $40 \%$ population within 10-15 fs. Obviously, the initial distribution of the trajectories is changed with respect to the full picture with 150 trajectories: The $S_{1}$ is now the strongest populated state in the beginning, while $\mathrm{S}_{2}$ and $\mathrm{S}_{3}$ states are less populated and therefore hit zero population faster. However, the early population of the triplet states and the deactivation channels, and therefore, the main conclusions discussed in the main text are the same as in the broader range. The reason why the two sets of energy ranges give very similar dynamical results is based on the fact that the $\mathrm{S}_{1}$ state provides the most important channel for the ultrafast ISC observed in our simulations. The branching between the two main deactivation pathways from $\mathrm{S}_{1}$ (i.e. ISC towards $\mathrm{T}_{2}$ and following IC towards $\mathrm{T}_{1}$ or IC from $\mathrm{S}_{1}$ to $\mathrm{S}_{0}$ ) is similar in the complete range of initial conditions. Then, because $\mathrm{S}_{1}$ and $\mathrm{T}_{2}$ are nearly degenerate for many geometries, the smaller excess energy of the trajectories with limited excitation bandwidth does not influence the efficiency of the ISC channel.
Thus, the early population of the triplet states and the deactivation channels discussed in the main text are almost independent of the excitation energy range.

The deactivation pathways visualized in Fig. 2 are based on the number of surface hops that occurred between the different pairs of states in the molecular dynamics simulations. Since jumps can take place in two directions, the net amount of hops for a pair of states $i$ and $j$ can be calculated by subtracting the number of hops from state $i$ to $j$ from the number of hops from $j$ to $i$. The temporal evolution of this quantity is shown in Fig. S3. Panel a) depicts the pathways for internal conversion (IC) between the singlet states, panel b) the intersystem crossing (ISC) pathways between singlet and triplet states and panel c) the IC between the triplets. The curves can be fitted with exponential functions according to $N_{i}\left(1-e^{-t / \tau_{i}}\right)$ in order to get time constants $\tau_{i}$ for the respective pathways. These time constants are collected in Table S1 and are consistent with all the available data. However, due to the sensitivity of the time constants with the energy and the lack of dynamical correlation in the electronic structure calculations, they should be regarded as qualitative. 


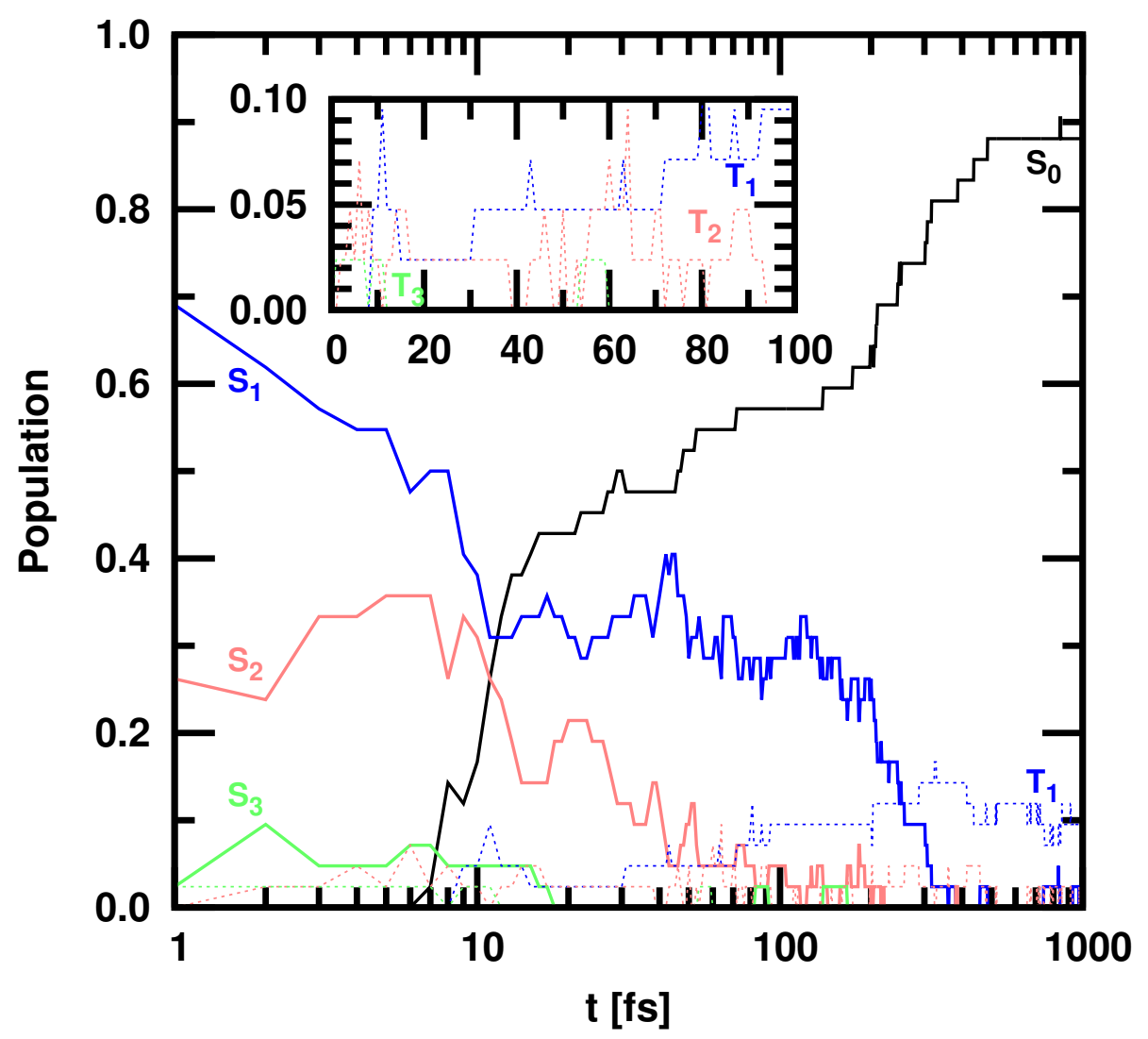

Figure S2: Time evolution of the singlet (solid) and triplet (dotted) states during the first picosecond. The inset zooms the first $100 \mathrm{fs}$. The $\mathrm{S}_{0}$ ground state population is in black, $\mathrm{S}_{1} / \mathrm{T}_{1}$ states populations are in red, $\mathrm{S}_{2} / \mathrm{T}_{2}$ in blue and $\mathrm{S}_{3} / \mathrm{T}_{3}$ in green. Excitation bandwidth is limited to $4.75 \pm$ $0.25 \mathrm{eV}$.
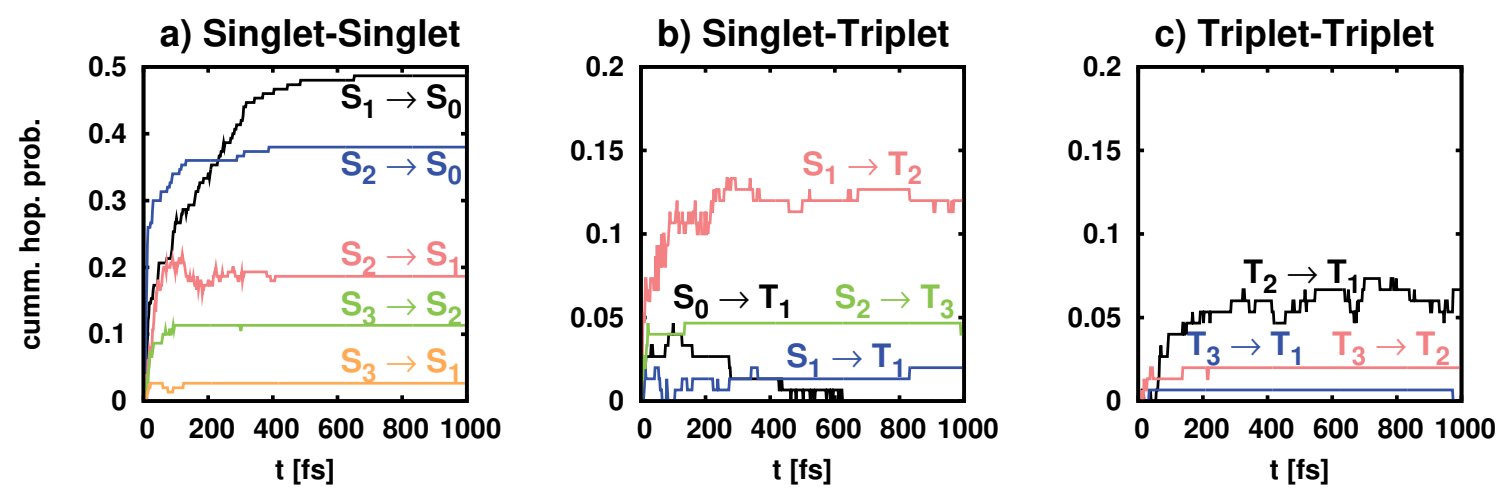

Figure S3: Net hops accumulated in time. 
Table S1: Time constants fitted to accumulated hopping probability curves (see Fig. S2)

\begin{tabular}{lll} 
Pathway & time constant & Error [\%] \\
\hline \hline $\mathrm{S}_{1} \rightarrow \mathrm{S}_{0}$ & $\tau_{1}=155 \mathrm{fs}$ & 1 \\
$\mathrm{~S}_{2} \rightarrow \mathrm{S}_{0}$ & $\tau_{2}=25 \mathrm{fs}$ & 2 \\
$\mathrm{~S}_{2} \rightarrow \mathrm{S}_{1}$ & $\tau_{3}=25 \mathrm{fs}$ & 3 \\
$\mathrm{~S}_{3} \rightarrow \mathrm{S}_{1}$ & $\tau_{4}=109 \mathrm{fs}$ & 3 \\
$\mathrm{~S}_{3} \rightarrow \mathrm{S}_{2}$ & $\tau_{5}=22 \mathrm{fs}$ & 2 \\
\hline $\mathrm{S}_{1} \rightarrow \mathrm{T}_{1}$ & $\tau_{6}=1000 \mathrm{fs}$ & $>100$ \\
$\mathrm{~S}_{1} \rightarrow \mathrm{T}_{2}$ & $\tau_{7}=78 \mathrm{fs}$ & 2 \\
$\mathrm{~S}_{2} \rightarrow \mathrm{T}_{3}$ & $\tau_{8}=14 \mathrm{fS}$ & 4 \\
\hline $\mathrm{T}_{2} \rightarrow \mathrm{T}_{1}$ & $\tau_{4}=216 \mathrm{fs}$ & 3 \\
$\mathrm{~T}_{3} \rightarrow \mathrm{T}_{1}$ & $\tau_{5}=33 \mathrm{fs}$ & 2 \\
$\mathrm{~T}_{3} \rightarrow \mathrm{T}_{2}$ & $\tau_{5}=82 \mathrm{fs}$ & 2
\end{tabular}




\section{Ab initio quantum chemical calculations}

At each time step of the propagation, the electronic states of keto-cytosine and corresponding electronic properties have been calculated using the state-average SA7-CAS $(12,9) / 6-31 G^{*}$ level of theory using 12 electrons distributed in 9 orbitals, as implemented in the MOLPRO software package. ${ }^{14}$ As in Ref., 16 the active space consists of $4 \pi, 3 \pi^{*}$ and $2 n$ orbitals, see Fig. S4. Seven states (four singlets and three triplets) are averaged with equal weigths. Relativistic corrections were taken into account by the use of the 2nd order Douglas-Kroll-Hess Hamiltonian. 20121

Table S2 shows the vertical excitation energies of the lowest-lying three singlet and triplet excited states at the equilibrium geometry obtained at $\operatorname{SA} 7-\operatorname{CASSCF}(12,9) / 6$ $31 \mathrm{G}^{*} / /$ B3LYP/TZVP level of theory as well as other levels of theory for comparison, obtained using MOLCAS 7.6. $22+24$ As it can be seen, the energies are rather sensitive to the introduction of dynamical correlation and also to the optimized geometry employed. The values obtained with $\operatorname{SA} 7-\operatorname{CASSCF}(12,9) / 6$ $31 \mathrm{G}^{*} / / \mathrm{B} 3 \mathrm{LYP} / \mathrm{TZVP}$ are similar to those obtained when calculating the singlets and triplet states separately (SA4+SA3). The energy of the lowest singlet state $\mathrm{S}_{1}$, which determines the maximum of the first UV absorption band, is decreased when perturbation theory is included (MS-CASPT2/SA7-CASSCF $(12,9) / 6$ $\left.31 \mathrm{G}^{*} / / \mathrm{B} 3 \mathrm{LYP} / \mathrm{TZVP}\right)$.

At MP2 level of theory the ground state equilibrium structure gets slightly pyramidalized at the $\mathrm{NH}_{2}$ group while B3LYP keeps the molecule completely planar. This geometrical difference is not relevant in the ab initio MD simulations, since an ensemble of geometries sampled over all the degrees of freedom according to the $\mathrm{ZPE}$ is considered. However, as it can be seen in Table S2, the out of plane deformation is important to obtain an energy closer to the experimental $\mathrm{S}_{1}$-origin in the gas phase $\mathrm{e}^{25126}$ located at ca. $4.7 \mathrm{eV}$. The MS-CASPT2 value for the $\mathrm{S}_{1}$ using SA4+SA3CAS $(12,9) / 6-31 \mathrm{G}^{*} / / \mathrm{MP} 2 / 6-31 \mathrm{G}^{*}$ level of theory is predicted at $4.8 \mathrm{eV}$, very close to the experimental one. A similar value is also obtained using DFT-MRCI calculations. ${ }^{27}$

Since MS-CASPT2 trajectories are computationally not affordable and electronic couplings are not available at DFT-MRCI level of theory, our calculations were done using SA7CAS $(12,9) / 6-31 G^{*} / /$ B3LYP/TZVP. The obtained vertical excitation energies are in agreement with those previously reported also at the CAS/6-31G** level of theory by Merchán and coworkers. $\stackrel{28}{ }$

\section{References}

(1) Richter, M.; Marquetand, P.; GonzálezVáquez, J.; Sola, I.; González, L. SHARC: Ab Initio Molecular Dynamics with Surface Hopping in the Adiabatic Representation Including Arbitrary Couplings. J. 


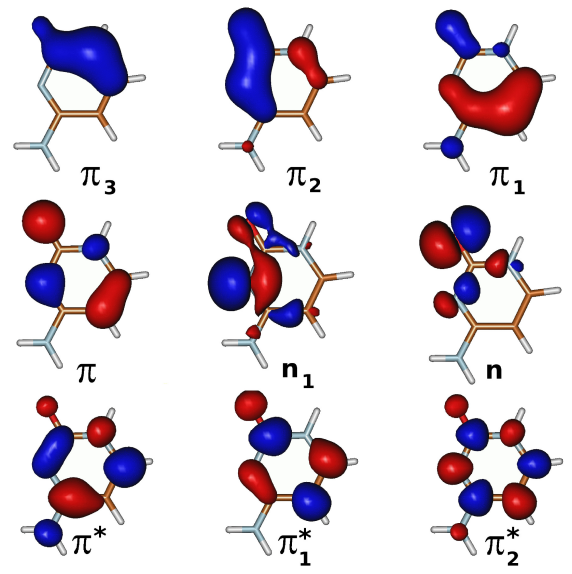

Figure S4: Orbitals included in the SA7-CASSCF $(12,9) / 6-31 G^{*}$ calculations.

Table S2: Vertical excitation energies of keto-cytosine at the equilibrium geometry computed at different levels of theory, as specified. Oscillator strengths are given in parentheses.

\begin{tabular}{c||ccc|ccc}
$a b$ initio methodolody & $\mathrm{S}_{1}$ & $\mathrm{~S}_{2}$ & $\mathrm{~S}_{3}$ & $\mathrm{~T}_{1}$ & $\mathrm{~T}_{2}$ & $\mathrm{~T}_{3}$ \\
\hline \hline SA7-CAS(12,9)/6-31G*//B3LYP/TZVP & $5.37(0.081)$ & $5.38(0.002)$ & $5.79(0.002)$ & 3.81 & 5.14 & 5.17 \\
SA4+SA3-CAS(12,9)/6-31G*//B3LYP/ZVP & $5.26(0.083)$ & $5.41(0.001)$ & $5.72(0.004)$ & 3.80 & 4.96 & 5.02 \\
MS-CASPT2 using previous one & $5.00(0.073)$ & $5.37(0.002)$ & $5.81(0.002)$ & 4.15 & 5.17 & 5.29 \\
\hline SA7-CAS(12,9)/6-31G*//MP2/6-31G* & $5.08(0.074)$ & $5.09(0.000)$ & $5.46(0.005)$ & 3.54 & 4.91 & 4.93 \\
SA4+SA3-CAS(12,9)/6-31G*//MP2/6-31G* & $4.98(0.080)$ & $5.10(0.001)$ & $5.45(0.004)$ & 3.48 & 4.74 & 4.77 \\
MS-CASPT2 using previous one & $4.80(0.072)$ & $5.15(0.002)$ & $5.61(0.002)$ & 3.96 & 4.90 & 5.17 \\
\hline \hline CAS(12,9)/6-31G** Ref & 5.32 & 5.34 & 5.67 & 3.72 & 5.01 & 5.19 \\
MS-CASPT2 Ref & $4.53(0.065)$ & $5.04(0.001)$ & $5.11(0.003)$ & 3.65 & 4.68 & 4.77 \\
\hline \hline DFT-MRCI//B3LYP/TZVP Ref & $4.83(0.080)$ & $5.02(0.002)$ & $5.50(0.001)$ & & & \\
\hline \hline
\end{tabular}

Chem. Theory. Comput. 2011, 7, 12531258 .

(2) Tully, J. C. Molecular Dynamics with Electronic Transitions. J. Chem. Phys. 1990, 93, 1061-1071.

(3) Newton, I. Philosophiae Naturalis Principia Mathematica 1726 (Havard Univ. Press, Cambridge).

(4) Verlet, L. Computer "Experiments" on Classical Fluids. II. Equilibrium Correlation Functions. Phys. Rev. 1968, 165, 201-214.

(5) Butcher, J. A Modified Multistep Method for the Numerical Integration of Ordinary Differential Equations. J. Assoc. Comput. Mach. 1965, 12, 124-135.
(6) Granucci, G.; Persico, M. Critical Appraisal of the Fewest Switching Algorithm for Surface Hopping. J. Chem. Phys. 2007, 126, 134114.

(7) Ahlrichs, R.; Bär, M.; Häser, M.; Horn, H.; Kölmel, C. Electronic Structure Calculations on Workstation Computers: The Program System Turbomole. Chem. Phys. Lett. 1989, 162, 165-169.

(8) Treutler, O.; Ahlrichs, R. Efficient Molecular Numerical Integration Schemes. J. Chem. Phys. 1995, 102, 346-354.

(9) Becke, A. D. Density-Functional Thermochemistry. III. The Role of Exact Exchange. J. Chem. Phys. 1993, 98, 56485652 .

(10) Lee, C.; Yang, W.; Parr, R. G. LYP Correlation: Development of the Colle-Salvetti 
Correlation-Energy Formula into a Functional of the Electron Density. Phys. Rev. B 1988, 37, 785 .

(11) Schäfer, A.; Huber, C.; Ahlrichs, R. Fully Optimized Contracted Gaussian Basis Sets of Triple Zeta Valence Quality for Atoms Li to Kr. J. Chem. Phys. 1994, 100, 5829-5835.

(12) Werner, H. J.; Knowles, P. J. A Second Order Multiconfiguration SCF Procedure with Optimum Convergence. J. Chem. Phys. 1985, 82, 5053-5063.

(13) Knowles, P. J.; Werner, H. J. An Efficient Second-Order MCSCF Method for Long Configuration Expansions. Chem. Phys. Lett. 1985, 115, $259-267$.

(14) Werner, H. J.; Knowles P. J.; Lindh, R.; Manby, F. R.; Schütz M.; Celani, P.; Korona, T.; Mitrushenkov, A.; Rauhut, G.; Adler, T. B. et al. MOLPRO, Version 2010.1, a Package of Ab Initio Programs.

(15) Barbatti, M.; Aquino, A. J. A.; Lischka, H. The UV Absorption of Nucleobases: Semi-Classical Ab Initio Spectra Simulations. Phys. Chem. Chem. Phys. 2010, 12, 4959-4967.

(16) González-Vázquez, J.; González, L. A Time-dependent Picture of the Ultrafast Deactivation of Keto-Cytosine Including Three-State Conical Intersections. Chem. Phys. Chem. 2010, 11, 3617-3624.

(17) Barbatti, M.; Granucci G.; Persico, M.; Ruckenbauer, M.; Vazdar, M.; EckertMaksić, M.; Lischka, H. The On-TheFly Surface-Hopping Program System Newton-X: Application to Ab Initio Simulation of the Nonadiabatic Photodynamics of Benchmark Systems. J. Photochem. Photobiol. A 2007, 190, 228-240.

(18) Barbatti, M.; Granucci, G.; M. Ruckenbauer, J. P.; Persico, M.; Lischka, H. Newton-X: A Package for Newtonian Dynamics Close to the Crossing Seam 2007, www.newtonx.org.
(19) Kosma, K.; Schröter, C.; Samoylova, E.; Hertel, I. V.; Schultz, T. Excited-State Dynamics of Cytosine Tautomers. J. Am. Chem. Soc. 2009, 131, 16939-16943.

(20) Douglas, M.; Kroll, N. M. Quantum Electrodynamical Corrections to the Finestructure of Helium. Ann. Phys. 1974, 82, 89-155.

(21) Hess, B. A. Relativistic ElectronicStructure Calculations Employing a Two-Component No-Pair Formalism with External-Field Projection Operators. Phys. Rev. A 1986, 33, 3742-3748.

(22) Aquilante, F.; Vico, L. D.; Ferré, N.; Ghigo, G.; Malmqvist, P.- $\AA$; Neogrády, P.; Pedersen, T. B.; Pitonak, M.; Reiher, M.; Roos, B. O. et al. MOLCAS 7: The Next Generation. J. Comp. Chem. 2010, 31, 224-247.

(23) Veryazov, V.; Widmark, P.; SerranoAndrés, L.; Lindh, R.; Roos, B. O. MOLCAS as a Development Platform for Quantum Chemistry Software. Int. J. Quantum Chem. 2004, 100, 626635.

(24) Karlstram, G.; Lindh, R.; Malmqvist, P.$\AA$; Roos, B. O.; Ryde, U.; Veryazov, V.; Widmark, P.-O.; Cossi, M.; Schimmelpfennig, M.; Neogrády, P.; Seijo, L. MOLCAS: A Program Package for Computational Chemistry. Comp. Mater. Sci. 2003, 28, 222-239.

(25) Nir, E.; Müller, M.; Grace, L.; de Vries, M. Rempi Spectroscopy of Cytosine. Chem. Phys. Lett. 2002, 355, $59-64$.

(26) Nir, E.; Plützer, C.; Kleinermanns, K.; de Vries, M. Properties of Isolated DNA Bases, Base Pairs and Nucleosides Examined by Laser Spectroscopy. Euro. Phys. J. D 2002, 20, 317-329.

(27) Tomić, K.; Tatchen, J.; Marian, C. M. Quantum Chemical Investigation of the Electronic Spectra of the Keto, Enol, and Keto-Imine Tautomers of Cytosine. J. Phys. Chem. A 2005, 109, 8410-8418. 
(28) Merchán, M.; Serrano-Andrés, L.; Robb, M. A.; Blancafort, L. Triplet-State Formation Along the Ultrafast Decay of Excited Singlet Cytosine. J. Am. Chem. Soc. 2005, 127, 1820-1825. 